Case Report

\title{
Macroscopic Hematuria due to Placenta Percreta: Report of Two Cases and Short Review
}

\author{
Ourania Koukoura, ${ }^{1}$ George Lialios, ${ }^{1}$ Antonios Garas, ${ }^{1}$ \\ George Sveronis, ${ }^{1}$ Asterios Nidimos, ${ }^{1}$ Irondiana Gkorezi, ${ }^{1}$ Zoi Alevra, ${ }^{1}$ Vassilios Tzortzis, ${ }^{2}$ \\ Athanasios Oeconomou, ${ }^{2}$ Ioannis Zachos, ${ }^{2}$ and Alexandros Daponte ${ }^{1}$
}

${ }^{1}$ Department of Obstetrics and Gynecology, University Hospital of Larissa, Larissa, Thessaly, Greece

${ }^{2}$ Department of Urology, University Hospital of Larissa, Larissa, Thessaly, Greece

Correspondence should be addressed to Ourania Koukoura; okoukoura@yahoo.com

Received 8 January 2017; Accepted 7 February 2017; Published 18 June 2017

Academic Editor: Giampiero Capobianco

Copyright (C) 2017 Ourania Koukoura et al. This is an open access article distributed under the Creative Commons Attribution License, which permits unrestricted use, distribution, and reproduction in any medium, provided the original work is properly cited.

\begin{abstract}
Herein we present two cases of pregnant women with placenta percreta and severe hematuria during the 24th and 35th weeks of pregnancy, respectively. A timely sonographic diagnosis was feasible in the first case and cesarean section was performed during the 29th week. During the operation, the placenta was invading the bladder wall and concomitant hysterectomy with cystotomy and bladder wall reconstruction was performed. The second case presented in our emergency department with vaginal bleeding during the 35th weeks of pregnancy. She underwent an emergency cesarean section with uterine preservation, cystotomy, and bladder reconstruction.
\end{abstract}

\section{Introduction}

Placenta percreta represents a rare pregnancy complication where the placenta invades the uterine myometrium and occasionally adjacent organs, such as the bladder. The prevalence of all types of abnormally invasive placenta (accreta, increta, and percreta) has been increased due to the rise in the cesarean section rate. It is estimated that invasive placenta cases have risen to 10 times over the last 50 years. Approximately $1 / 500$ to $1 / 2500$ deliveries are complicated by invasive placenta [1]. Placenta percreta is associated with significant morbidity. When placenta percreta is invading the bladder, mortality rates may be as high as $9.5 \%$ and $24 \%$ for mother and baby, respectively [2]. Herein we report two cases of placenta percreta with bladder invasion, one of which was diagnosed prenatally during the second trimester of pregnancy. In the second case, the diagnosis was made antenatally during the 35 th weeks of pregnancy immediately prior to the emergency cesarean section.

\section{Case Presentation}

2.1. Case 1. A 39-year-old woman (gravida 3, para 2) was referred to our department during the 24th week of pregnancy due to gross hematuria. The pregnancy was uncomplicated till then; however the diagnosis of complete placenta previa was made during the anatomic scan three weeks prior to her admission. She had two previous cesarean sections and no other medical history. An abdominal sonographic evaluation revealed a viable fetus with appropriate biometrical parameters and normal amniotic fluid, while transvaginal scan suggested the presence of complete placenta previa with bladder wall invasion (Figure 1). She was admitted to our department for expectant management. A course of steroids was administered to promote fetal lung maturation. On hospital day \#1, bladder irrigation was performed by the urologists and a Foley catheter was placed in order to control hematuria. The patient had intermittent macroscopic hematuria and cystoscopy was performed on day \#4 in order to confirm the diagnosis; however the examination was 


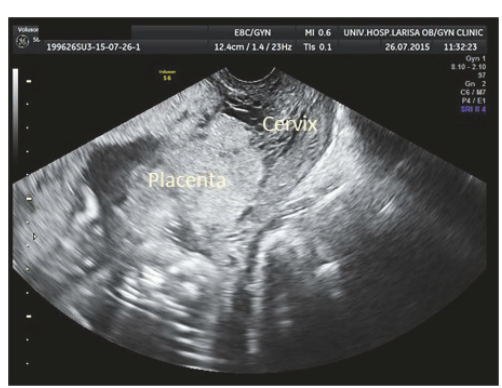

(a)

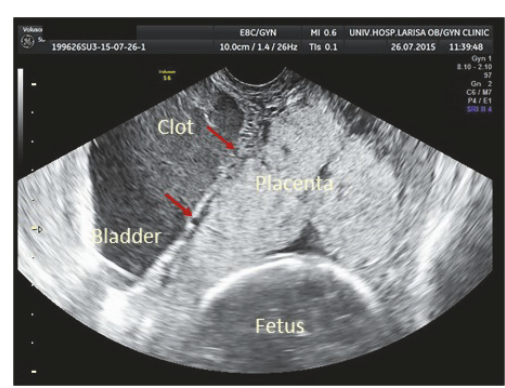

(b)

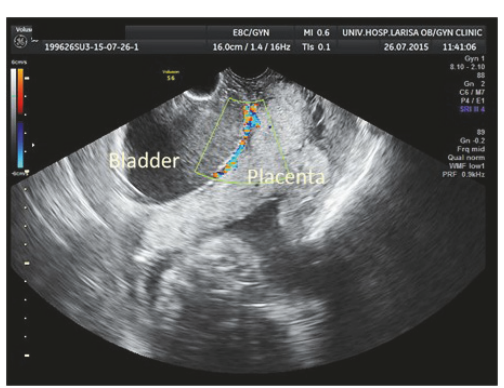

(c)

Figure 1: (a) Complete placenta previa. (b) Disruption of the bladder-placental interface indicated by red arrows. (c) Color Doppler revealed hypervascularity between the placenta and the bladder.

inconclusive. On hospital day \#10, evaluation of the placenta with magnetic resonance imaging (MRI) was also nondiagnostic. The patient was asymptomatic by day \#20 and she was discharged from the hospital. Two days later, however, the woman became symptomatic and was then readmitted with hematuria and vaginal bleeding. She had a hematocrit of $\mathrm{Ht} 22.1 \%$ and hemoglobin $\mathrm{Hb} 6.8 \mathrm{mg} / \mathrm{dL}$ and was transfused with two units of blood. The patient went into labor and she was immediately transferred to the operating room. A multidisciplinary team by obstetricians, midwifes, urologists, neonatologists, and anesthetists was on site. A viable male baby, weighing $1000 \mathrm{gr}$, was delivered by cesarean section and concomitant abdominal hysterectomy. The baby was transferred to the NICU where he was intubated. Dissection of the placenta from the anterior uterine wall created a large defect on the dome of the bladder. The bladder was repaired with two layers of continuous Vicryl 0 suture. A Foley catheter and an intraperitoneal drain were placed. The patient required transfusion of 20 units of various blood products intraoperatively. The postoperative course was uneventful and the patient was discharged on the 10th postoperative day. She made a good recovery; however urologic evaluation one month postoperatively revealed a vesicovaginal fistula, which was surgically treated at a later date. The baby was discharged three months after delivery.

2.2. Case 2. A 26-year-old woman (gravida 4, para 3) presented in our emergency department with vaginal bleeding during the 35th week of pregnancy. She had three previous cesarean sections and no antenatal screening during the present pregnancy. She was transferred to the labor ward where appropriate for gestational age embryo and anterior placenta previa detected during abdominal ultrasound scan. An emergency cesarean section was scheduled and just prior to the operation a Foley catheter was inserted to the bladder which drained blood. The cesarean section was carried out with a vertical incision high in the anterior uterine wall. A male baby weighing 2250 gr was delivered with Apgar scores of 8 and 9 and was transferred to the NICU for further evaluation. Interrupted Vicryl 1 sutures were used for closure of the uterine wall. Intraoperative bleeding was moderate and no transfusion was necessary at that point. A urologist evaluated and repaired the bladder defect which was away from both ureteral orifices. The patient made an unremarkable recovery and both she and the baby were discharged on the 5th postoperative day. The Foley catheter was removed on the 10th postoperative day.

\section{Discussion}

The exact incidence of placenta percreta remains unclear, since the invasiveness of the placenta is not always established, making it difficult to distinguish between placenta percreta, increta, and accreta. Earlier reports describe the incidence of placenta percreta as within 1:1000 to $1: 70,000$ births [3]. Placenta percreta represents the most severe form of placental invasiveness which requires aggressive evaluation and management to decrease morbidity. Multiparity, prior uterine surgery, most commonly cesarean section, and advanced maternal age have been all identified as risk factors for developing this complication of pregnancy [4]. There is a linear increase in the incidence of invasive placenta with the number of previous cesarean deliveries. Diagnosis should always be suspected in any pregnancy with previous uterine scar and detection of a low-lying placenta or placenta previa. It is estimated that when the detection of placenta previa is made in a woman with three previous cesarean sections, there is a $40 \%$ chance of the occurrence of placenta accrete [5]. Both cases in our report had previous cesarean sections and, in the first case, the diagnosis of placenta previa was established during the 21st week's anatomic scan.

Prenatal diagnosis of placenta percreta can help reduce maternal/fetal morbidity and mortality by allowing us to choose the best time and place of delivery. Specific sonographic criteria have been recommended for a timely diagnosis of morbidly adherent placenta. Detailed medical history and high index of suspicion is essential in these cases. The sonographic features include an inability to visualize the normal retroplacental clear zone, irregularity of the serosa-bladder interface, intraplacental lacunar spaces, and hypervascularity between the placenta and the bladder when using the color Doppler, resembling a large aneurysm [69]. Transvaginal scan was indicative of placental invasion in our first case, since there was a distinct disruption of the bladder wall (Figure 1(b)). We employ the use of magnetic resonance imaging (MRI) to confirm diagnosis. Magnetic 
resonance imaging (MRI) has similar performance status to the ultrasound; however it can be helpful in cases where placenta is difficult to visualize on ultrasound due to the patient's high body mass index. Expertise in such cases is often required to obtain an accurate diagnosis [10]. Although sonographic evaluation revealed a high suspicion of placental invasion in our first case, MRI was nondiagnostic and final diagnosis of bladder invasion was confirmed intraoperatively.

Hematuria is a rare occurrence comprising $25 \%$ of all placenta percreta cases [11]. Although not a sensitive finding, placenta percreta with bladder invasion should be suspected in any pregnant woman presenting with gross hematuria and a history of previous cesarean sections [12]. Placental bladder infiltration is a potentially life-threatening situation and therefore mandates a multidisciplinary surgical management, adequate number of blood products, neonatal intensive care, and the option of uterine artery embolization if needed. Hysterectomy without any attempts to remove the placenta is the recommended option [13]. Separation of the bladder from the placenta may cause severe blood loss. Even in cases with major intraoperative bleeding, bladder integrity preservation should be one of the primary surgical goals. Cesarean section and hysterotomy can be carried out through the bladder incision whenever this is unavoidable. Preoperative placement of ureteric stents is essential to minimize ureteral injury.

Another more recent optional management involves the placenta to be left in situ during cesarean delivery and postoperative methotrexate injection [14, 15]. Concomitant vascular embolization minimizes intraoperative blood loss and may facilitate postoperative placental involution. In such cases, regular postpartum monitoring is required, since complete regression of the placental remnants may take several months to ensue. None of our cases were treated conservatively. Although diagnosis in the first of our cases was made several weeks prior to the operation, emergency cesarean section and hysterectomy were performed based on the sudden deterioration of the patient's condition. In the second case, cesarean section was performed with a high vertical incision and no attempts to separate the bladder were made. Blood loss was controllable and hence hysterectomy was avoided [16]. Bladder was repaired with two layers of sutures in both cases. Potential long-term consequences of bladder damage include fistulas, urinary incontinence, and sexual dysfunction.

Patients with suspected placenta percreta with bladder invasion should be counseled about the potential risks of all management options. The surgical plan should be documented and alternative treatments should be available. The woman should become aware that efforts to preserve her uterus might increase morbidity and that planned hysterectomy is a safer option than peripartum hysterectomy.

\section{Conflicts of Interest}

No potential conflicts of interest were reported by the authors.

\section{References}

[1] S. Wu, M. Kocherginsky, and J. U. Hibbard, "Abnormal placentation: twenty-year analysis," American Journal of Obstetrics and Gynecology, vol. 192, no. 5, pp. 1458-1461, 2005.

[2] F. V. Price, E. Resnik, K. A. Heller, and W. A. Christopherson, "Placenta previa percreta involving the urinary bladder: A report of two cases and review of the literature," Obstetrics and Gynecology, vol. 78, no. 3, pp. 508-511, 1991.

[3] R. Pohl, "The special case: rare gynecologic emergency of uterine perforation in placenta percreta," Fortschritte der Medizin, vol. 97, no. 46, pp. 2143-2144, 1979.

[4] R. M. Silver and K. D. Barbour, "Placenta Accreta Spectrum. Accreta, Increta, and Percreta," Obstetrics and Gynecology Clinics of North America, vol. 42, no. 2, pp. 381-402, 2015.

[5] R. M. Silver, M. B. Landon, D. J. Rouse et al., "Maternal morbidity associated with multiple repeat cesarean deliveries," Obstetrics and Gynecology, vol. 107, no. 6, pp. 1226-1232, 2006.

[6] H. J. Finberg and J. W. Williams, "Placenta accreta: Prospective sonographic diagnosis in patients with placenta previa and prior cesarean section," Journal of Ultrasound in Medicine, vol. 11, no. 7, pp. 333-343, 1992.

[7] D. M. Twickler, M. J. Lucas, A. B. Balis et al., "Color flow mapping for myometrial invasion in women with a prior cesarean delivery," The Journal of Maternal-Fetal Medicine, vol. 9, no. 6, pp. 330-335, 2000.

[8] C. H. Comstock, "The antenatal diagnosis of placental attachment disorders," Current Opinion in Obstetrics and Gynecology, vol. 23, no. 2, pp. 117-122, 2011.

[9] G. P. Guy, D. B. Peisner, and I. E. Timor-Tritsch, "Ultrasonographic evaluation of uteroplacental blood flow patterns of abnormally located and adherent placentas," American Journal of Obstetrics and Gynecology, vol. 163, no. 3, pp. 723-727, 1990.

[10] C. H. Comstock and R. A. Bronsteen, "The antenatal diagnosis of placenta accreta," BJOG: An International Journal of Obstetrics and Gynaecology, vol. 121, no. 2, pp. 171-181, 2014.

[11] R. Konijeti, J. Rajfer, and A. Askari, "Placenta percreta and the urologist," Rev Urol, vol. 11, no. 3, pp. 173-176, 2009.

[12] F. Abbas, J. Talati, S. Wasti, S. Akram, S. Ghaffar, and R. Qureshi, "Placenta percreta with bladder invasion as a cause of life threatening hemorrhage," Journal of Urology, vol. 164, no. 4, pp. 1270-1274, 2000.

[13] R. M. Silver, K. A. Fox, and J. R. Barton, "Center of excellence for placenta accreta," American Journal of Obstetrics \& Gynecology, vol. 212, no. 5, pp. 561-568, 2015.

[14] M. Khan, P. Sachdeva, R. Arora, and S. Bhasin, "Conservative management of morbidly adherant placenta - A case report and review of literature," Placenta, vol. 34, no. 10, pp. 963-966, 2013.

[15] S. K. Doumouchtsis and S. Arulkumaran, "The morbidly adherent placenta: an overview of management options," Acta Obstetricia et Gynecologica Scandinavica, vol. 89, no. 9, pp. 11261133, 2010.

[16] O. Koukoura, G. Lialios, A. Garas et al., "Macroscopic hematuria due to placenta percreta. Report of two cases. Oral presentation," in Proceedings of the 10th Athens Congress on Womens Health and Disease, Athens, Greece, September 2016. 


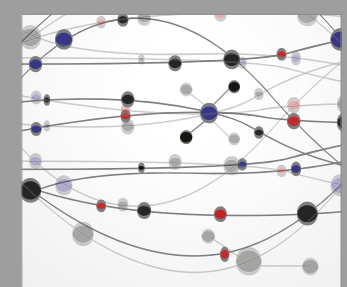

The Scientific World Journal
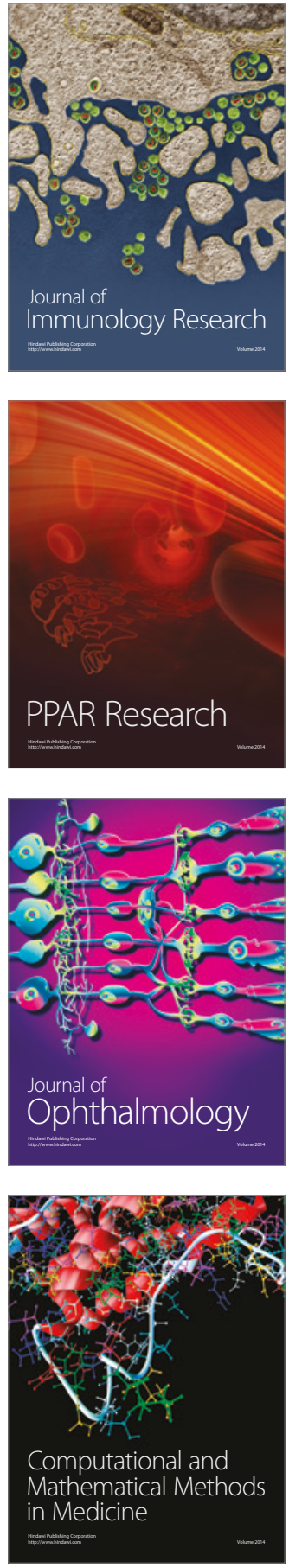

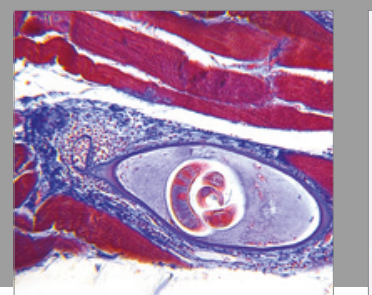

Gastroenterology Research and Practice
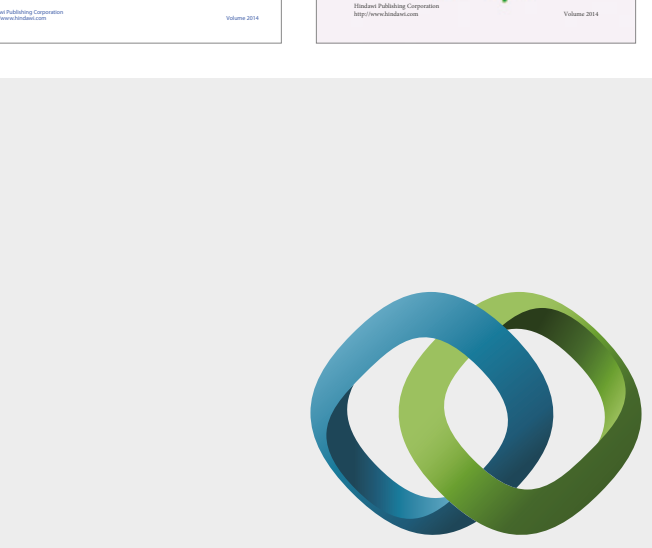

\section{Hindawi}

Submit your manuscripts at

https://www.hindawi.com
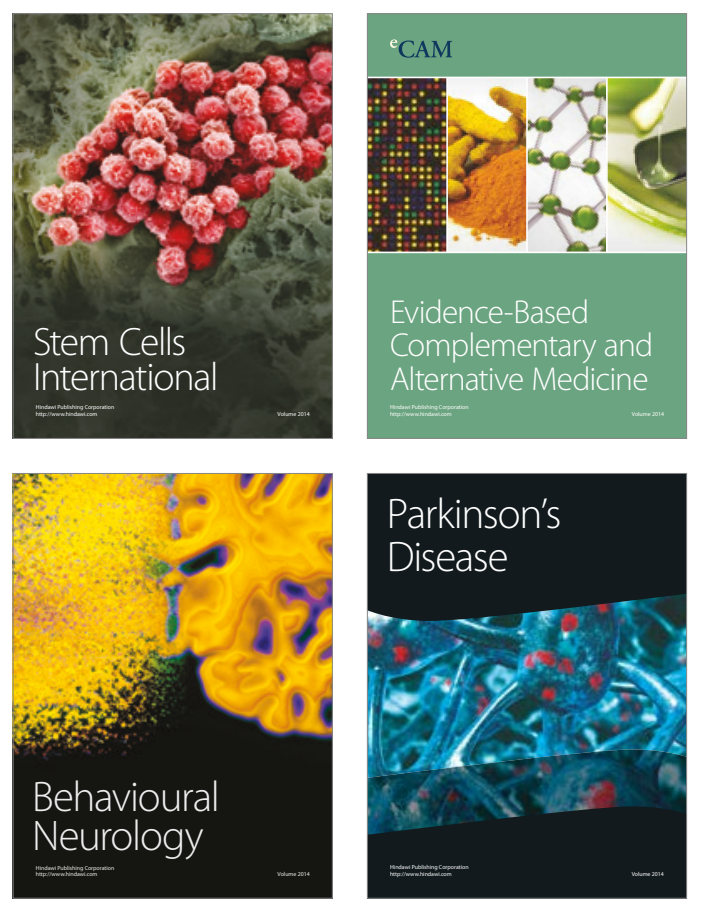
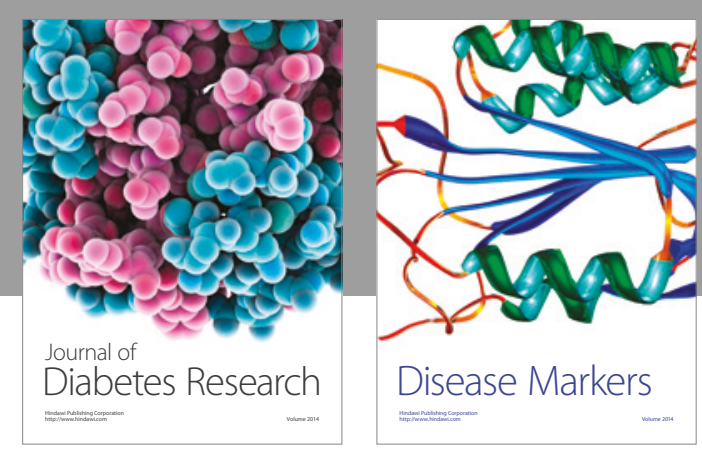

Disease Markers
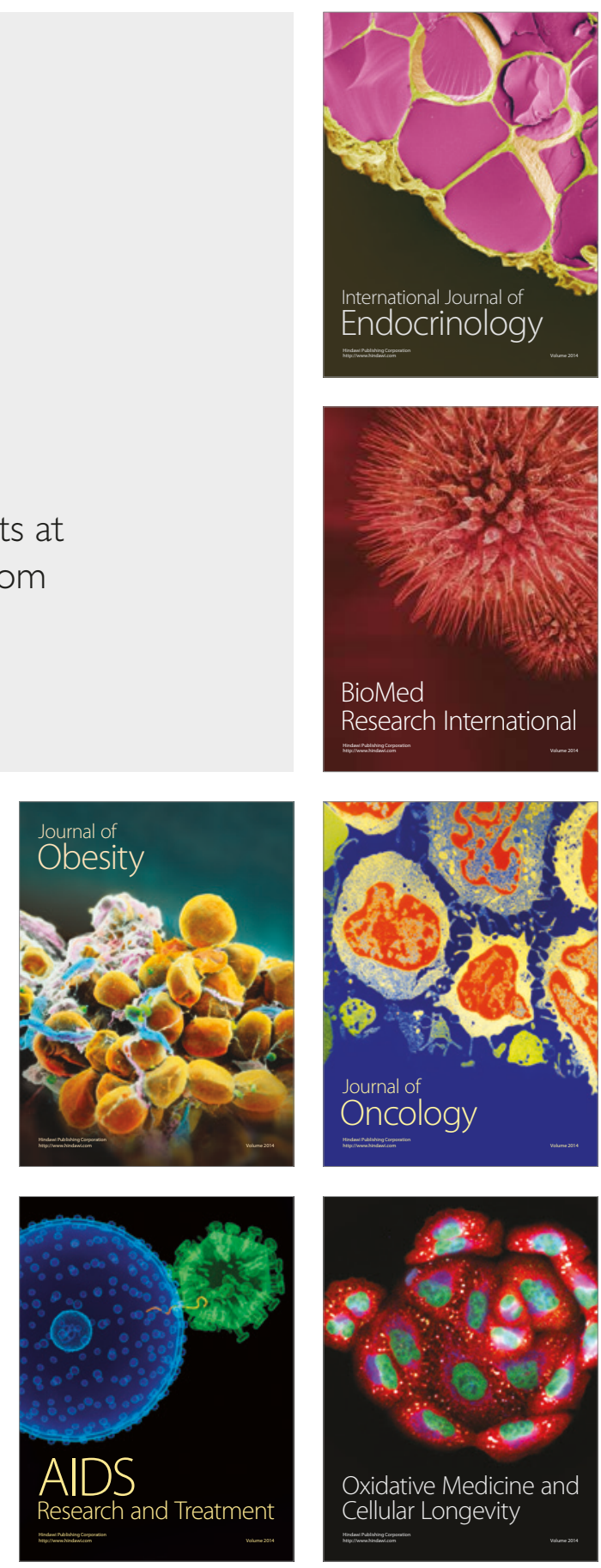